\title{
Finding the Order in Complexity: The Electronic Structure of 14-1-11 Zintl Compounds
}

\author{
Yukun Liu* ${ }^{1}$ Michael Y. Toriyama* ${ }_{1}^{1}$ Zizhen Cai, ${ }^{1}$ Mengjia Zhao, ${ }^{1}$ Fei Liu, ${ }^{1}$ and G. \\ Jeffrey Snyder ${ }^{\ddagger 1}$ \\ Department of Materials Science and Engineering, Northwestern University, \\ Evanston, IL, USA, 60208
}

(Dated: 10 October 2021)

$\mathrm{Yb}_{14} \mathrm{MnSb}_{11}$ and $\mathrm{Yb}_{14} \mathrm{MgSb}_{11}$ have rapidly risen to prominence as high-performing p-type thermoelectric materials for potential deep space power generation. However, the fairly complex crystal structure of 14-1-11 Zintl compounds renders the interpretation of the electronic band structure obscure, making it difficult to chemically guide band engineering and optimization efforts. In this work, we delineate the valencebalanced Zintl chemistry of $A_{14} M X_{11}$ compounds ( $A=\mathrm{Yb}, \mathrm{Ca} ; M=\mathrm{Mg}, \mathrm{Mn}, \mathrm{Al}$, $\mathrm{Zn}, \mathrm{Cd} ; X=\mathrm{Sb}, \mathrm{Bi}$ ) using molecular orbital theory analysis. By analyzing the electronic band structures of $\mathrm{Yb}_{14} \mathrm{MgSb}_{11}$ and $\mathrm{Yb}_{14} \mathrm{AlSb}_{11}$, we show that the conduction band minimum is composed of either an antibonding molecular orbital originating from the $\left(\mathrm{Sb}_{3}\right)^{7-}$ trimer, or a mix of atomic orbitals of $A, M$, and $X$. The singly degenerate valence band is comprised of non-bonding $\mathrm{Sb}_{\mathrm{z}}$ orbitals primarily from the $\mathrm{Sb}$ atoms in the $\left(\mathrm{MSb}_{4}\right)^{\mathrm{m}-}$ tetrahedra and the of isolated $\mathrm{Sb}$ atoms distributed throughout the unit cell. Such a chemical understanding of the electronic structure enables strategies to engineer electronic properties (e.g., the band gap) of $A_{14} M X_{11}$ compounds.

\footnotetext{
$1 *$ Y.L. and M.Y.T. contributed equally to this work.

$\ddagger$ jeff.snyder@northwestern.edu
} 
Thermoelectric materials can reversibly and directly convert heat into electricity with no moving parts. However, the energy conversion efficiencies of current thermoelectric devices remain insufficient for widespread applications. Thermoelectric energy conversion efficiency is thermodynamically limited by the Carnot efficiency and depends on the material figure of merit $\left(z T=\alpha^{2} \sigma T / \kappa\right)^{1}$. Conceptually, to obtain a large $z T$, a large Seebeck coefficient $(\alpha)$ and electrical conductivity $(\sigma)$ are desired, while the thermal conductivity $(\kappa)$ should be minimized. However, because these properties are highly interrelated and all good thermoelectric materials behave as heavily doped semiconductors ${ }^{2}$, engineering $z T$ is best understood as optimizing the charge carrier concentration $n$ and maximizing the thermoelectric quality factor $B \sim \mu_{\mathrm{W}} / \kappa_{\mathrm{L}}$ where $\mu_{\mathrm{W}}$ is the weighted mobility ${ }^{3}$ and $\kappa_{\mathrm{L}}$ is the lattice thermal conductivity.

The weighted mobility depends on the electronic structure through the effective mass and valley degeneracy but also the electron scattering. Generally, a complex band structure with high effective valley degeneracy is desired ${ }^{4}$ while flat bands with high band effective mass are detrimental to mobility ${ }^{5,6}$. In addition, the character of the bands can help determine how strong a substitutional atom will scatter electrons or holes by how much it alters the conduction or valence band.

The optimal charge carrier concentration is engineered by intrinsic defects and extrinsic substitutional atoms where an understanding of the Zintl chemistry is essential ${ }^{7}$.

The discovery ${ }^{8}$ of the complex Zintl compound, $\mathrm{Yb}_{14} \mathrm{MnSb}_{11}$ as a high $z T$, hightemperature $(900 K-1300 K)$, p-type thermoelectric material has paved the way for $20 \%$ or more efficient thermoelectric power generation such that it has been selected by NASA for next-generation radioisotope thermoelectric generators (RTGs) for space missions ${ }^{9}$. With improved processing, and various chemical substitutions of the $A$ or $M$ atom in $A_{14} M \mathrm{Sb}_{11}$ several examples with $z T>1$ have been reported ${ }^{10}$. All of the compounds have exceptionally low lattice thermal conductivity $\left(\kappa_{\mathrm{L}}\right)$ because of the large unit cell ${ }^{11}$ that limits propagating acoustic waves to a small frequency range and so most of the heat is transported by diffusons ${ }^{12}$.

Despite the complex structure, a number of chemical variations are known with the $A_{14} M X_{11}$ structure (Figure 1), many with similar electronic properties. ${ }^{13}$ The $A$ atom is generally a +2 cation such as $\mathrm{Yb}^{14,15}, \mathrm{Ca}^{15,16}$, and $\mathrm{Eu}^{17,18}$ where +3 Rare Earth elements (e.g. $\mathrm{La}^{19}$ and $\mathrm{Y}^{20}$ ) can be substituted. The $M$ atom can be a variety of transition metals 


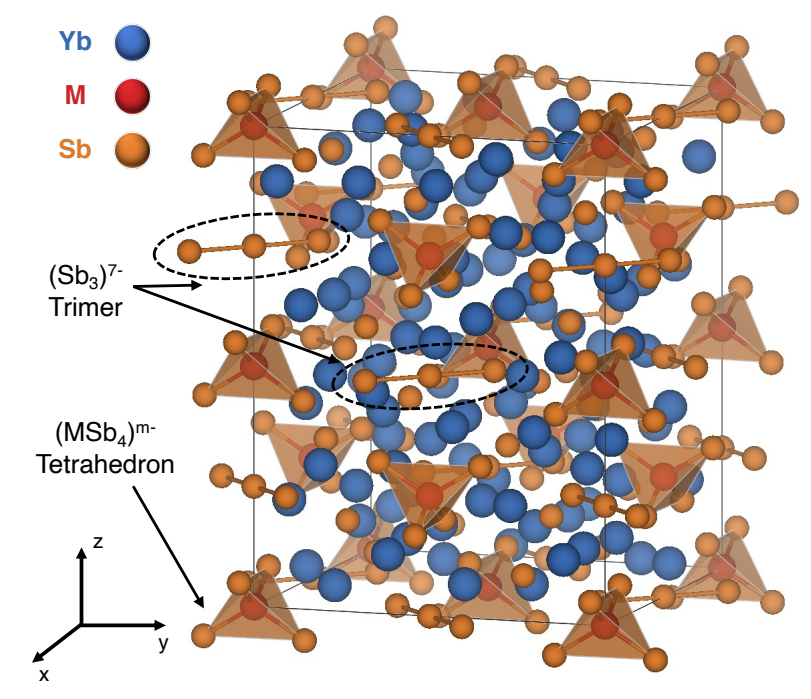

FIG. 1. The unit cell of $\mathrm{Yb}_{14} M \mathrm{Sb}_{11}(M=\mathrm{Mg}, \mathrm{Mn}, \mathrm{Al}, \mathrm{Zn}$, or $\mathrm{Cd})$. Yb atoms are indicated by blue spheres, $\mathrm{Sb}$ atoms are brown, $\mathrm{Sb}_{3}$ linear units are shown as bonded brown atoms, and $\left(M \mathrm{Sb}_{4}\right)^{\mathrm{m}-}$ are indicated by brown tetrahedra where $\mathrm{m}$ is 9 for $M=\mathrm{Mn}, \mathrm{Al}$ and 10 for $M=\mathrm{Mg}, \mathrm{Zn}, \mathrm{Cd}$.

suitable for centering the $M X_{4}$ tetrahedron ( The $X$ atom can be any of the pnictides $\left(\mathrm{Sb}^{15}, \mathrm{As}^{24}\right.$ or $\left.\mathrm{Bi}^{16}\right)$.

From detailed experimental and theoretical analysis of the high $z T$ compositions $\mathrm{Yb}_{14} \mathrm{MnSb}_{11}$ and $\mathrm{Yb}_{14} \mathrm{MgSb}_{11}$ and related compounds it has been shown ${ }^{15,23,25-28}$ that the valence bands are essentially the same for the Sb compounds. The valence bands contain a simple, light valence band with a second heavier valence band close in energy that has high valley degeneracy which provides additional conduction paths, contributing to a high electrical conductivity, while maintaining a large thermopower ${ }^{5,6}$.

The electronic structure and chemical tunability of $\mathrm{Yb}_{14} M \mathrm{Sb}_{11}$ can be generally explained using Zintl Chemistry ${ }^{7}$. Zintl compounds can be described as a network of covalently bonded complex anions with charge balanced by cations donating their valence electrons ${ }^{29}$. Thus, the valence of an atom can be defined as follows:

$$
\begin{gathered}
V_{c}=e_{c}-b_{c} \\
V_{a}=e_{a}+b_{a}-8
\end{gathered}
$$

where $V$ represents the valence, $e$ is the number of valence electrons, and $b$ is the number 
of formed bonds, while $c$ and $a$ denote cations and anions, respectively. Each bond between anions or between cations reduces the magnitude of the valence by 1. Utilizing this charge counting method, the complex structure of $\mathrm{Yb}_{14} M \mathrm{Sb}_{11}$ can be described as consisting of $\left(M \mathrm{Sb}_{4}\right)^{m-}(m=9$ or 10$)$ isolated tetrahedra, $\left(\mathrm{Sb}_{3}\right)^{7-}$ linear units, and isolated $\mathrm{Yb}^{2+}$ and $\mathrm{Sb}^{3-}$ ions. The value of $m$ depends on the valence of the atom on the $M$ site. When $M$ is trivalent, like $\mathrm{Al}^{3+}, m=9$ and the compound is a valence balanced semiconductor. When $M$ is divalent, like $\mathrm{Mg}^{2+}, m=10$ and the compounds are slightly electron poor making a p-type metal or heavily doped semiconductor. This system of charge counting has enabled tuning of the free charge carrier concentration in $A_{14} M \mathrm{Sb}_{11}$ compounds by substituting some $A$ and $M$ atoms with others of a different valence ${ }^{30,31}$

Another way to understand the chemistry of the Zintl compounds is to combine the Zintl concept with molecular orbital analysis ${ }^{32}$. This method has been successfully applied to halfHeusler compounds to interpret band structures based on the characteristics of molecular orbitals ${ }^{33}$. The high symmetry of the clearly distinguished $\left(M \mathrm{Sb}_{4}\right)^{m-}$ and $\left(\mathrm{Sb}_{3}\right)^{7-}$ structural units renders possibilities of identifying their molecular orbitals in the electronic structure to interpret the complex band structures.

In this study, we will focus on interpreting the computed band structures of two $\mathrm{Yb}_{14} M \mathrm{Sb}_{11}$ compounds: $\mathrm{Yb}_{14} \mathrm{MgSb}_{11}$ and $\mathrm{Yb}_{14} \mathrm{AlSb}_{11}$ using the Zintl concept in combination with a molecular orbital analysis. We show that the molecular orbitals for structural units $\left(M \mathrm{Sb}_{4}\right)^{m-}$, and $\left(\mathrm{Sb}_{3}\right)^{7-}$ are easily identified giving clear indication as to which atoms dominate the conduction and valence bands. We discuss how this method can be used to understand and modify the electronic properties of $A_{14} M X_{11}$ compounds providing strategies to optimize their thermoelectric performance. All first-principles Density Functional Theory (DFT) calculations were performed used the Vienna ab-initio simulation package (VASP) $)^{34-39}$ using the projector-augmented wave (PAW) method $^{40,41}$ and an energy cutoff of $500 \mathrm{eV}$. The $4 \mathrm{f}$ electrons of $\mathrm{Yb}$ were not treated explicitly as valence electrons following the methodology of Ref. 25. The structures were relaxed with a force convergence criterion of $0.005 \mathrm{eV} / \AA$ with a $\Gamma$-centered $k$-point grid $^{42}$ of $3 \times 3 \times 3$. Static calculations were subsequently performed on the relaxed structures with a $k$-point grid of $5 \times 5 \times 5$ using the tetrahedron method ${ }^{43}$ for density of states calculations.

The electronic structures of $\mathrm{Yb}_{14} \mathrm{MgSb}_{11}$ and $\mathrm{Yb}_{14} \mathrm{AlSb}_{11}$ exhibit many overlapping bands (Figure 2), owing to the large number of atoms in the unit cells. Despite the complexity, the 
band structures reveal distinctive features based on the structural chemistry of the $\left(\mathrm{Sb}_{3}\right)^{7-}$ trimers and $\left(M \mathrm{Sb}_{4}\right)^{m-}$ tetrahedra.

The Fermi energy is at the valence band maximum when $M$ in $A_{14} M X_{11}$ is trivalent (e.g., $\mathrm{Al}^{3+}$, see Figure $2 \mathrm{~b}$ ), since there are 14 nonbonding $\mathrm{Yb}^{2+}$ atoms, 4 nonbonding $\mathrm{Sb}^{3-}$ atoms, a single $\left(M \mathrm{Sb}_{4}\right)^{9-}$ tetrahedral unit, and a single $\left(\mathrm{Sb}_{3}\right)^{7-}$ trimer per formula unit. The valence-balanced $\mathrm{Yb}_{14} \mathrm{AlSb}_{11}$ therefore exhibits semiconductor behavior. ${ }^{30}$ In contrast, the Fermi energy is buried in a partially-filled valence band when $M$ is divalent (e.g., $\mathrm{Mg}^{2+}$, see Figure 2a). $\mathrm{Yb}_{14} \mathrm{MgSb}_{11}$ is therefore deficient of one electron per formula unit, according to charge counting: $14 \mathrm{Yb}^{2+}+\left(\mathrm{MgSb}_{4}\right)^{10-}+\left(\mathrm{Sb}_{3}\right)^{7-}+4 \mathrm{Sb}^{3-}+1 \mathrm{~h}^{+}$. This indicates that the valence band is partially filled and explains why the compound exhibits behaviors of a heavily doped semiconductor. ${ }^{29}$

The conduction bands are largely dominated by Yb-s states, and one can therefore expect the energies of the conduction bands to be affected by the electronegativity of the $A$ cation in $A_{14} M X_{11}$. The unoccupied conduction bands are also mixed with Sb-p and Al-p character from the $\left(\mathrm{Sb}_{3}\right)^{7-}$ trimers and $\left(M \mathrm{Sb}_{4}\right)^{m-}$ tetrahedra. Notably, there are four distinctly low-energy conduction bands that are isolated from the rest of the conduction bands in $\mathrm{Yb}_{14} \mathrm{MgSb}_{11}$, which we call the $\sigma^{*}$ bands (Figure 3a). These bands form a narrow peak in the DOS $\sim 1 \mathrm{eV}$ above the Fermi energy (Figure $2 \mathrm{a}$ ) and are primarily of $\left(\mathrm{Sb}_{3}\right)^{7-}$ character. The charge density distribution of the $\sigma^{*}$ bands (Figure 3a) shows that the bands are antibonding states of the $\left(\mathrm{Sb}_{3}\right)^{7-}$ trimer unit. The antibonding character is evidenced by the nodal features of the charge density, where the wave function is zero between the middle $\mathrm{Sb}$ atom and the ends of the trimer, owing to the destructive interference of p-orbitals aligned along the axis of the trimer unit.

The $\sigma^{*}$ bands are critical in that they resolve differences in the conduction band structures of 14-1-11 compounds. For example, we can identify the same $\sigma^{*}$ bands in $\mathrm{Yb}_{14} \mathrm{AlSb}_{11}$ (Figure $3 \mathrm{~b}$ ), only they are not isolated from the rest of the conduction bands in this compound. This difference can be attributed to the $M$ cation in $A_{14} M X_{11}$. In $\mathrm{Yb}_{14} M \mathrm{Sb}_{11}$ compounds, the conduction bands are separated into those that are purely of $\left(\mathrm{Sb}_{3}\right)^{7-}$ character (i.e., the $\sigma^{*}$ bands) and those that are higher in energy. The latter group of conduction bands have partial $M$ character, meaning that the bands will be lower in energy in $\mathrm{Yb}_{14} \mathrm{AlSb}_{11}$ than in $\mathrm{Yb}_{14} \mathrm{MgSb}_{11}$ due to the higher electronegativity of Al. We also do not expect the absolute energies of the $\sigma^{*}$ bands to differ significantly between the two compounds, as the $\left(\mathrm{Sb}_{3}\right)^{7-}$ 
(a) $\mathrm{Yb}_{14} \mathrm{MgSb}_{11}$
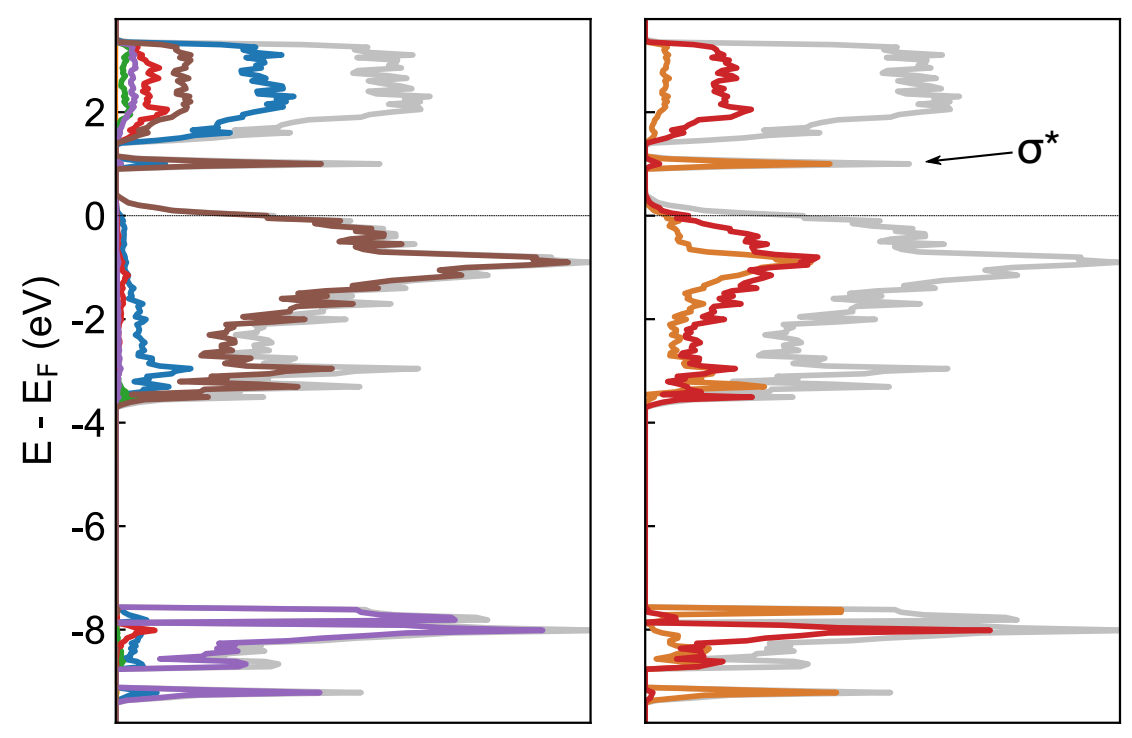

(b) $\mathrm{Yb}_{14} \mathrm{AlSb}_{11}$

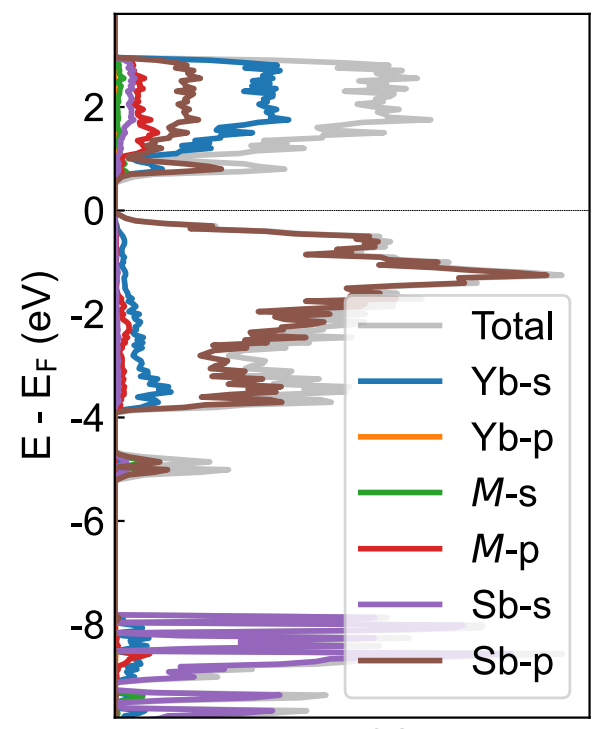

Density of States

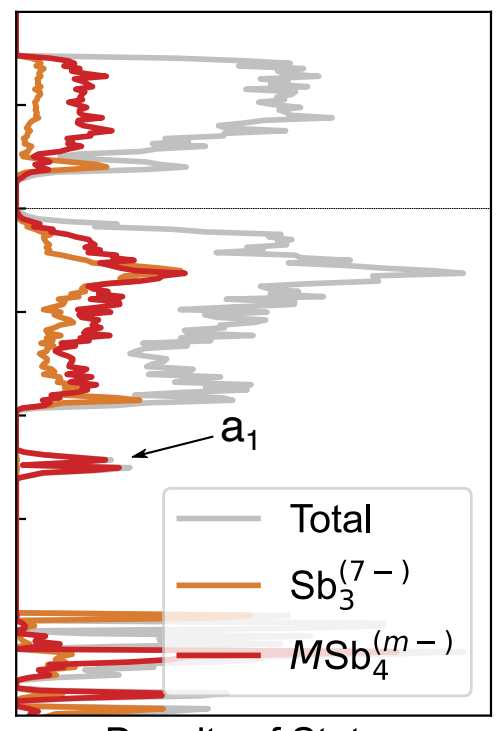

Density of States

FIG. 2. Density of states of (a) $\mathrm{Yb}_{14} \mathrm{MgSb}_{11}$ and (b) $\mathrm{Yb}_{14} \mathrm{AlSb}_{11}$. The partial density of states of the $\left(\mathrm{Sb}_{3}\right)^{7-}$ trimers, and the $M \mathrm{Sb}_{4}$ tetrahedra are also shown. The $\sigma^{*}$ states are labeled in the $\mathrm{Yb}_{14} \mathrm{MgSb}_{11}$ panel, and the $\mathrm{a}_{1}$ states are labeled in the $\mathrm{Yb}_{14} \mathrm{AlSb}_{11}$ panel.

trimer units exist in both compounds. It is as if the higher-energy conduction bands of $\mathrm{Yb}_{14} \mathrm{AlSb}_{11}$ have "descended" in energy, merging with the $\sigma^{*}$ bands unlike in $\mathrm{Yb}_{14} \mathrm{MgSb}_{11}$.

The valence bands on the other hand are primarily of Sb character, with the p-orbitals contributing to the bands from $\sim 4 \mathrm{eV}$ below the Fermi energy up to the valence band 

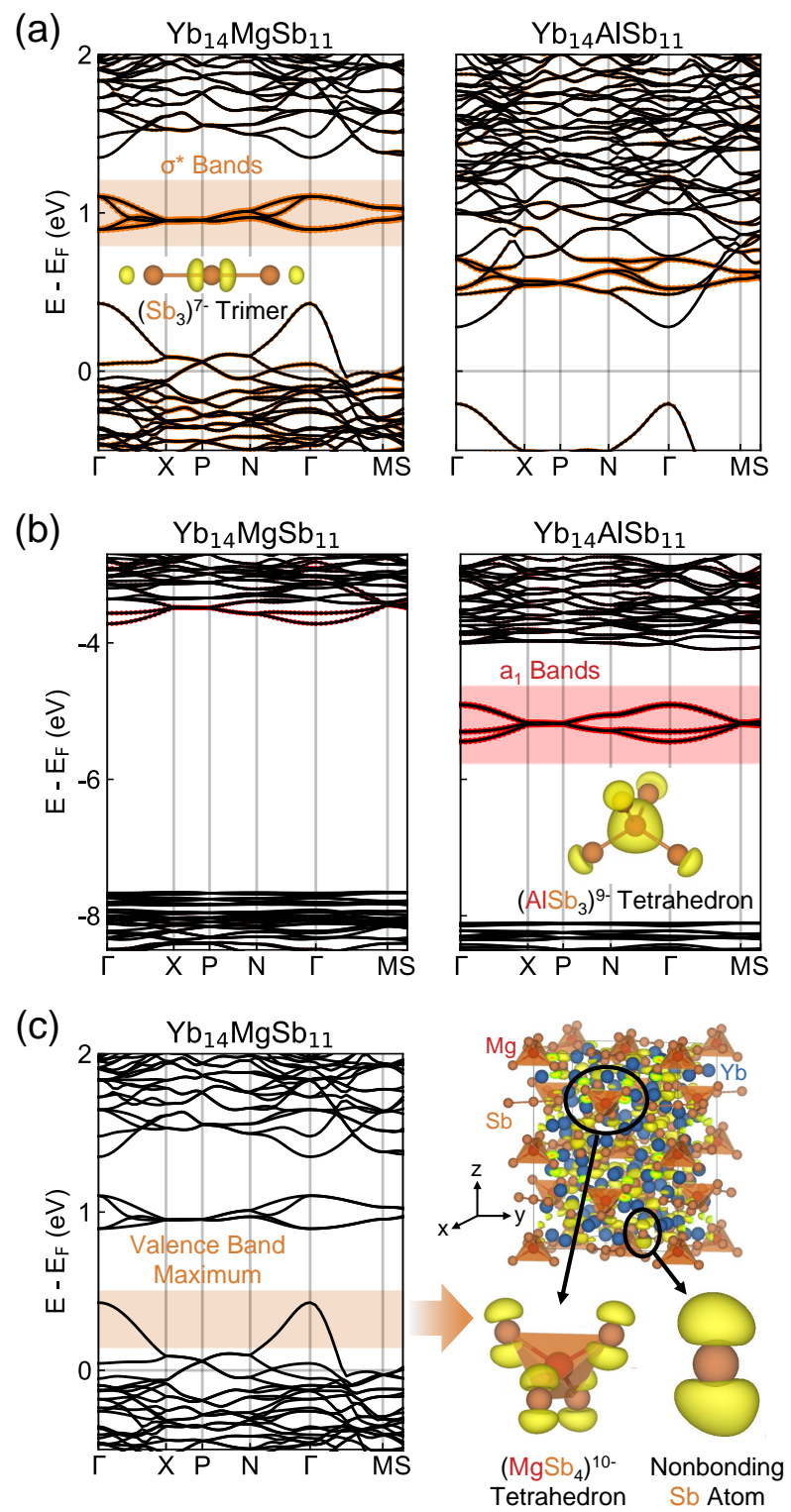

FIG. 3. (a) Band structures of $\mathrm{Yb}_{14} \mathrm{MgSb}_{11}$ and $\mathrm{Yb}_{14} \mathrm{AlSb}_{11}$, where the size of the orange circles correspond to the relative contributions from the $\left(\mathrm{Sb}_{3}\right)^{7-}$ trimers to the bands. The charge density of $\mathrm{Yb}_{14} \mathrm{MgSb}_{11}$ within the orange highlighted portion of the band structure is localized only on the $\left(\mathrm{Sb}_{3}\right)^{7-}$ trimers, as shown for one trimer unit. (b) Band structures plotted within a lower energy range, where contributions from the $\mathrm{Mg} / \mathrm{Al}$ atoms are shown as red circles. The charge density of $\mathrm{Yb}_{14} \mathrm{AlSb}_{11}$ within the pink highlighted portion is localized only on the $\left(\mathrm{AlSb}_{4}\right)^{9-}$ tetrahedra, as shown for one unit. (c) The band structure of $\mathrm{Yb}_{14} \mathrm{MgSb}_{11}$, highlighting the energy window of the valence band for which the charge density is calculated. The charge density near the valence band maximum is dominated by the nonbonding $\mathrm{Sb} \mathrm{p}_{\mathrm{z}}$ orbitals primarily on the $\left(\mathrm{MgSb}_{4}\right)^{10-}$ tetrahedra and nonbonding Sb atoms, with some contribution from the trimer. 
maximum $(\mathrm{VBM})$ and the s-orbitals contributing to the bands located $\sim 8 \mathrm{eV}$ below the Fermi energy (Figure 2). Similar to the $\sigma^{*}$ conduction bands, we can distinguish features of the valence bands between $\mathrm{Yb}_{14} \mathrm{AlSb}_{11}$ and $\mathrm{Yb}_{14} \mathrm{MgSb}_{11}$. Notably, an isolated set of peaks appears in the DOS $\sim 5 \mathrm{eV}$ below the Fermi energy in $\mathrm{Yb}_{14} \mathrm{AlSb}_{11}$ that is not present in $\mathrm{Yb}_{14} \mathrm{MgSb}_{11}$. There are four bands that contribute to the peaks (the $\mathrm{a}_{1}$ bands), which are mainly comprised of $\mathrm{Al}-\mathrm{s}$ and $\mathrm{Sb}-\mathrm{p}$ atomic orbitals in $\mathrm{Yb}_{14} \mathrm{AlSb}_{11}$, as evidenced by both the partial density of states (Figure $2 \mathrm{~b}$ ) and the orbital-decomposed band structure (Figure 3b). Moreover, the charge density distribution of the $\mathrm{a}_{1}$ bands (Figure $3 \mathrm{~b}$ ) suggests that the states are of bonding character. While the $\mathrm{a}_{1}$ bands are isolated from the rest of the valence bands in $\mathrm{Yb}_{14} \mathrm{AlSb}_{11}$, orbital contributions from $\mathrm{Mg}$ indicates that the $\mathrm{a}_{1}$ bands are merged into the other valence bands in $\mathrm{Yb}_{14} \mathrm{MgSb}_{11}$ (Figure 3b). The difference in the electronic structures can again be attributed to the higher electronegativity of $\mathrm{Al}$, which lowers the energies of the $\mathrm{a}_{1}$ bands in $\mathrm{Yb}_{14} \mathrm{AlSb}_{11}$ relative to those in $\mathrm{Yb}_{14} \mathrm{MgSb}_{11}$.

The valence band maximum is a singly-degenerate, relatively light band which emerges from the flatter valence bands at the $\Gamma$ point (Figure 3c). The charge density of this band exhibits $\mathrm{p}_{\mathrm{z}}$-type orbitals centered on the $\mathrm{Sb}$ atoms of the $\left(M \mathrm{Sb}_{4}\right)^{m-}$ tetrahedra and nonbonding Sb atoms (Figure 3c) with less contribution from the trimer. The fact that only the $\mathrm{p}_{\mathrm{z}}$ orbitals of $\mathrm{Sb}$ atoms make up the light valence band suggests that hole transport should be somewhat anisotropic with different electrical conductivity in the $x$ and $y$ directions. We note that the valence bands in $A_{14} M S b_{11}$ compounds are quite similar, with a single dispersive band centered at the $\Gamma$ point and flatter, non-dispersive bands below the band extremum (see e.g., $\mathrm{Yb}_{14} \mathrm{MnSb}_{11}{ }^{25}$ ). The fact that these bands are dominated by Sb-atoms explains why the p-type thermoelectric properties of all $A_{14} M \mathrm{Sb}_{11}$ compounds are similar for the same hole concentration.

Despite the complex electronic structure of $\mathrm{Yb}_{14} M \mathrm{Sb}_{11}$, many distinguishing features can be explained using molecular orbital theory ${ }^{44}$ and Zintl chemistry. ${ }^{32}$ The structural makeup of $\mathrm{Yb}_{14} M \mathrm{Sb}_{11}$ compounds consists of $\left(\mathrm{Sb}_{3}\right)^{7-}$ trimers, $\left(M \mathrm{Sb}_{4}\right)^{m-}$ tetrahedra, isolated $\mathrm{Sb}$ anions, and isolated $\mathrm{Yb}$ cations. These components generate bands at different energy ranges due to the energies of the atomic orbitals and bonding/antibonding interactions.

The $\left(\mathrm{Sb}_{3}\right)^{7-}$ linear trimer unit is isoelectronic to the triiodide ion $\mathrm{I}_{3}^{-}$and therefore has similar molecular orbitals (Figure 4a). ${ }^{24,32,45}$ The relative phases of the Sb-p orbitals in $\left(\mathrm{Sb}_{3}\right)^{7-}$ lead to distinct molecular orbital energies based on whether the neighboring interactions are 
bonding, antibonding, or nonbonding, as well as their configurations (i.e., whether they are $\sigma$ - or $\pi$-oriented, see Figure 4a). When the p-orbitals of all three $\mathrm{Sb}$ atoms align in-phase along the axis of the trimer unit, the lowest-energy $\sigma$ bonding state will form. However, if the neighboring interactions are out-of-phase instead, then the highest-energy $\sigma^{*}$ antibonding state will form. Due to the linear structure of the $\left(\mathrm{Sb}_{3}\right)^{7-}$ trimer, there is only one $\sigma$ bonding state and one $\sigma^{*}$ antibonding state. Additionally, the p-orbitals of the Sb atoms can align perpendicular to the axis of the trimer unit, forming doublet $\pi$ bonding and doublet $\pi^{*}$ antibonding states. The $\pi$ and $\pi^{*}$ states are closer in energy than the $\sigma$ and $\sigma^{*}$ states, as $\pi$-bonds are typically weaker than $\sigma$-bonds ${ }^{32}$. Moreover, there are nonbonding states both in the $\sigma$ and $\pi$ configurations. In total, each $\left(\mathrm{Sb}_{3}\right)^{7-}$ trimer unit contributes nine bands (two $\sigma$-type, four $\pi$-type, and three nonbonding) to the electronic structure of $\mathrm{Yb}_{14} M \mathrm{Sb}_{11}$, of which eight are filled with two electrons each. The one unfilled state, the $\sigma^{*}$ molecular orbital, should appear in the conduction bands of $\mathrm{Yb}_{14} M \mathrm{Sb}_{11}$. As a matter of fact, the $\sigma^{*}$ molecular orbital in Figure 4a corresponds precisely to the $\sigma^{*}$ bands in Figure 3a: the four $\sigma^{*}$ bands come from the four $\left(\mathrm{Sb}_{3}\right)^{7-}$ trimer units per primitive cell of $\mathrm{Yb}_{14} M \mathrm{Sb}_{11}$, and the charge density distribution of the $\sigma^{*}$ bands indicate antibonding character.

The molecular orbital diagram of the $\left(\mathrm{AlSb}_{4}\right)^{9-}$ tetrahedral unit (Figure $4 \mathrm{~b}$ ) is constructed following the methodology of Toriyama et al. ${ }^{46}$ The bonding $\mathrm{a}_{1}$ state of $\left(\mathrm{AlSb}_{4}\right)^{9-}$ is a molecular orbital in which the $\mathrm{a}_{1}$ state of the $\mathrm{Sb}_{4}$ complex, which appears as dumbbell-like orbitals at each corner of the tetrahedron oriented towards the central $\mathrm{Al}$ atom, interferes constructively with the s-orbital of the central Al atom. Due to the constructive interference, we expect the charge density for the $\mathrm{a}_{1}$ state to be high between the central $\mathrm{Al}$ atom and the surrounding $\mathrm{Sb}_{4}$ complex, as in the charge density shown in Figure $3 \mathrm{~b}$. On the other hand, the destructive interference leads the formation of $a_{1}^{*}$ state. Moreover, we expect from molecular orbital theory that the $\mathrm{a}_{1}$ bonding state of $\left(\mathrm{AlSb}_{4}\right)^{9-}$ consists of Al-s, Sb-s, and Sb-p character by symmetry, all of which are reflected in the $a_{1}$ peaks in the orbitaldecomposed DOS in Figure $2 \mathrm{~b}$. As a result, the $\mathrm{a}_{1}$ bands in $\mathrm{Yb}_{14} M \mathrm{Sb}_{11}$ correspond exactly to the $\mathrm{a}_{1}$ bonding state shown in Figure $4 \mathrm{~b}$ as expected from molecular orbital theory, where we have four $\mathrm{a}_{1}$ bands since there are four $\left(M \mathrm{Sb}_{4}\right)^{m-}$ tetrahedral units per primitive cell.

A chemical intuition for the electronic structures of $\mathrm{Yb}_{14} \mathrm{MgSb}_{11}$ and $\mathrm{Yb}_{14} \mathrm{AlSb}_{11}$ described here can guide band engineering efforts of $A_{14} M X_{11}$ compounds in general, as similar electronic structures have been observed in this class of materials. For example, charge 
(a)
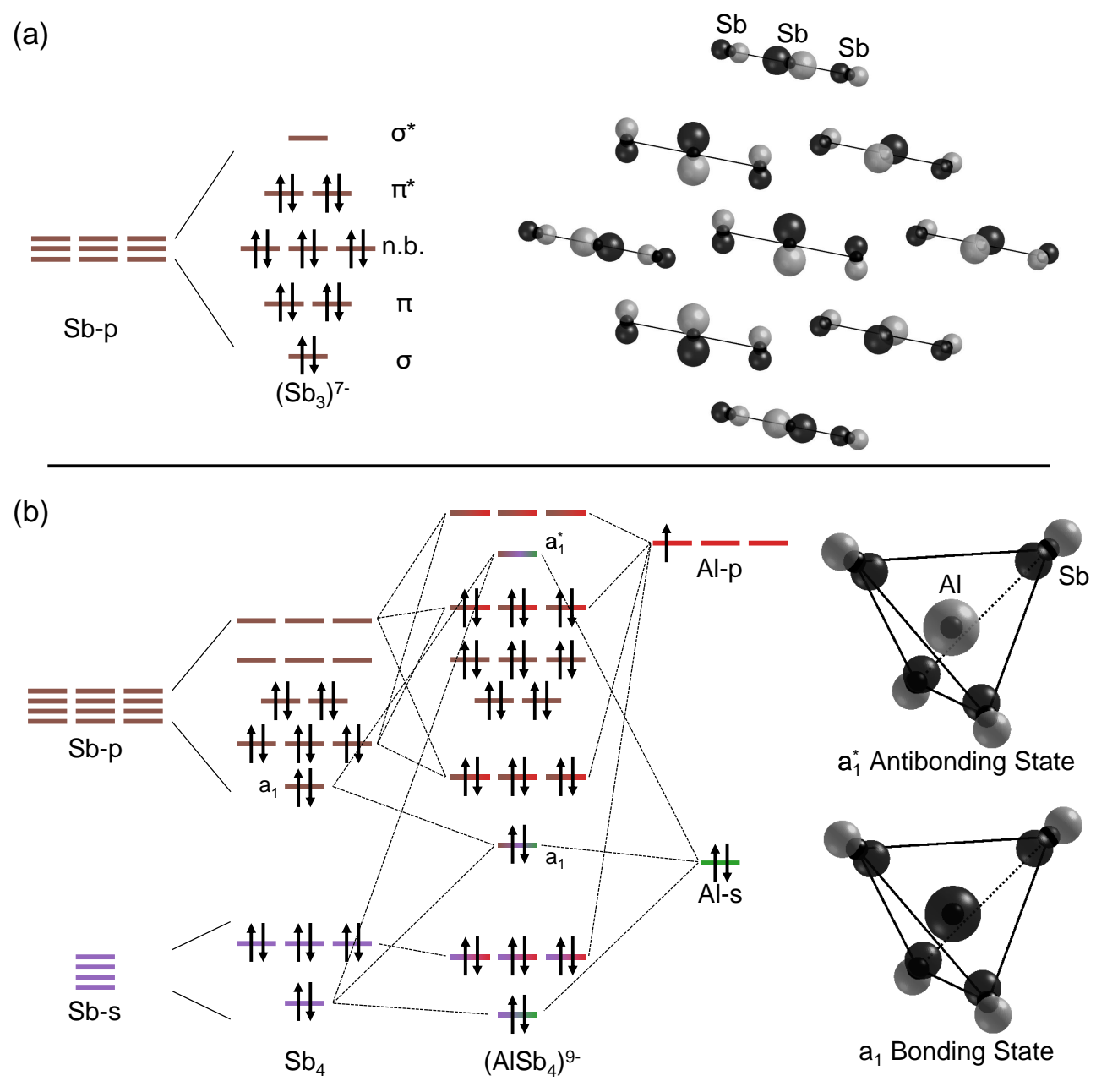

FIG. 4. (a) The molecular orbital diagram for a $\left(\mathrm{Sb}_{3}\right)^{7-}$ trimer unit in $A_{14} M \mathrm{Sb}_{11}$, where the molecular orbitals are constructed from the p-orbitals of the $\mathrm{Sb}$ atoms. The p-orbitals of the $\mathrm{Sb}$ atoms generate nine molecular orbitals in total, two of which are $\sigma$-type singlet states, four $\pi$ states, and a triplet set of nonbonding (n.b.) states. (b) The molecular orbital diagram for the $\left(\mathrm{AlSb}_{4}\right)^{9-}$ tetrahedral unit of $\mathrm{Yb}_{14} \mathrm{AlSb}_{11}$. The $\mathrm{a}_{1}$ state generated by the Sb-p orbitals hybridizes with the s-orbital of $\mathrm{Al}$, forming a bonding $\mathrm{a}_{1}$ state and an antibonding $\mathrm{a}_{1}^{*}$ state. The shading of the molecular orbitals denotes the relative phases.

density contour plots ${ }^{24}$ of the $\left(\mathrm{As}_{3}\right)^{7-}$ trimer unit in $\mathrm{Ca}_{14} \mathrm{GaAs}_{11}$ are reminiscent of the molecular orbitals derived for the $\left(\mathrm{Sb}_{3}\right)^{7-}$ trimer in Figure $4 \mathrm{a}$. The antibonding $\sigma^{*}$ bands from the trimer unit is also observed in many compounds with the same structure type, regardless of whether the $\sigma^{*}$ bands are isolated from the other conduction bands $\left(\mathrm{Ca}_{14} \mathrm{MgSb}_{11}{ }^{13}\right.$ $\mathrm{Yb}_{14} \mathrm{MnSb}_{11},{ }^{25} \mathrm{Ca}_{14} \mathrm{MnBi}_{11},{ }^{45} \mathrm{Ca}_{14} \mathrm{MgBi}_{11},{ }^{47} \mathrm{Sr}_{14} \mathrm{ZnAs}_{11}{ }^{48}$ ) or merged into the higher-energy 
conduction bands $\left(\mathrm{Ca}_{14} \mathrm{AlBi}_{11},{ }^{16} \mathrm{Ba}_{14} \mathrm{MnBi}_{11},{ }^{45} \mathrm{Ca}_{14} \mathrm{ZnSb}_{11},{ }^{49} \mathrm{Sr}_{14} \mathrm{CdSb}_{11}{ }^{23}\right)$.

The conduction band minimum is determined by the relative energies of the $\sigma^{*}$ bands originating from the $\left(X_{3}\right)^{7-}$ trimers and the nonbonding $A$ orbitals, and the valence band maximum at the $\Gamma$ point is determined by the energies of the $\mathrm{p}_{\mathrm{z}}$ orbitals of $X$ atoms that are either nonbonding or involved in the $\left(M X_{4}\right)^{m-}$ tetrahedral units. This may explain the saturation in the measured Seebeck coefficient of $\mathrm{Yb}_{14-x} \mathrm{Pr}_{x} \mathrm{MnSb}_{11}$ with increasing $\mathrm{Pr}$ content, ${ }^{50}$ where the band gap remains unchanged past a certain Pr content likely due to the fixed energies of the Sb-derived band edges.

In summary, despite their complex structures, the electronic structures of $A_{14} M X_{11}$ compounds can be understood using Zintl chemistry in combination with molecular orbital theory. The derived bonding/antibonding interactions within the structural units successfully explain the electronic structures calculated using first-principles, building a chemical intuition for $A_{14} M X_{11}$ compounds.

\section{ACKNOWLEDGMENTS}

We thank Trinh Vo, Susan Kauzlarich and Paul von Allmen for preliminary work and helpful discussions. This work was supported by NSF DMREF award \#1729487. M.Y.T. is funded by the United States Department of Energy through the Computational Science Graduate Fellowship (DOE CSGF) under grant number DE-SC0020347. This research was supported in part through the computational resources and staff contributions provided for the Quest high performance computing facility at Northwestern University, which is jointly supported by the Office of the Provost, the Office for Research, and Northwestern University Information Technology.

\section{DATA AVAILABILITY}

The data that support the findings of this study are available from the corresponding author upon reasonable request. 


\section{AUTHOR DECLARATIONS}

\section{Conflict of interest}

The authors have no conflicts to disclose.

\section{REFERENCES}

${ }^{1}$ G. J. Snyder and E. S. Toberer, "Complex thermoelectric materials," Nat. Mater. 7, 105 (2008).

${ }^{2}$ X. Zhang, Z. Bu, X. Shi, Z. Chen, S. Lin, B. Shan, M. Wood, A. H. Snyder, L. Chen, G. J. Snyder, and Y. Pei, "Electronic quality factor for thermoelectrics," Science Advances 6, eabc0726 (2020).

${ }^{3}$ G. J. Snyder, A. H. Snyder, M. Wood, R. Gurunathan, B. H. Snyder, and C. Niu, "Weighted mobility," Adv. Mater. 32, 2001537 (2020).

${ }^{4}$ Z. M. Gibbs, F. Ricci, G. Li, H. Zhu, K. Persson, G. Ceder, G. Hautier, A. Jain, and G. J. Snyder, "Effective mass and fermi surface complexity factor from ab initio band structure calculations," npj Comput. Mater. 3, 1 (2017).

${ }^{5}$ Y. Pei, H. Wang, and G. J. Snyder, "Band engineering of thermoelectric materials," Adv. Mater. 24, 6125 (2012).

${ }^{6}$ Y. Pei, A. D. LaLonde, H. Wang, and G. J. Snyder, "Low effective mass leading to high thermoelectric performance," Energ. Environ. Sci. 5, 7963 (2012).

${ }^{7}$ E. S. Toberer, A. F. May, and G. J. Snyder, "Zintl chemistry for designing high efficiency thermoelectric materials," Chem. Mater. 22, 624 (2010).

${ }^{8}$ S. R. Brown, S. M. Kauzlarich, F. Gascoin, and G. J. Snyder, "Yb ${ }_{14} \mathrm{mnns}_{11}$ : New high efficiency thermoelectric material for power generation," Chem. Mater. 18, 1873 (2006).

${ }^{9}$ S. Kauzlarich and S. K. Bux, "Earth abundant high temperature materials for radioisotope power conversion system," Tech. Rep. (University of California, 2016).

${ }^{10}$ A. P. Justl, G. Cerretti, S. K. Bux, and S. M. Kauzlarich, "Hydride assisted synthesis

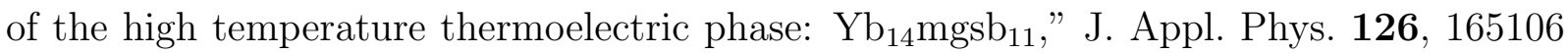
(2019).

${ }^{11}$ E. S. Toberer, A. Zevalkink, and G. J. Snyder, "Phonon engineering through crystal chemistry," J. Mater. Chem. 21, 15843-15852 (2011). 
${ }^{12}$ R. Hanus, J. George, M. Wood, A. Bonkowski, Y. Cheng, D. L. Abernathy, M. E. Manley, G. Hautier, G. J. Snyder, and R. P. Hermann, "Uncovering design principles for amorphous-like heat conduction using two-channel lattice dynamics," Mater. Today Phys. 18, 100344 (2021).

${ }^{13}$ Y. Hu, G. Cerretti, E. L. K. Wille, S. K. Bux, and S. M. Kauzlarich, "The remarkable crystal chemistry of the $\mathrm{ca}_{14} \mathrm{alsb}_{11}$ structure type, magnetic and thermoelectric properties," J. Solid State Chem. 271, 88 (2019).

${ }^{14} \mathrm{Y}$. Hu and S. M. Kauzlarich, "Yb ${ }_{14} \mathrm{mgbi}_{11}$ : structure, thermoelectric properties and the effect of the structure on low lattice thermal conductivity," Dalton T. 46, 3996 (2017).

${ }^{15}$ Y. Hu, J. Wang, A. Kawamura, K. Kovnir, and S. M. Kauzlarich, "Yb ${ }_{14} \mathrm{mgsb}_{11}$ and $\mathrm{ca}_{14} \mathrm{mgsb}_{11}$ - new mg-containing zintl compounds and their structures, bonding, and thermoelectric properties," Chem. Mater. 27, 343 (2015).

${ }^{16} \mathrm{~S}$. Baranets and S. Bobev, "Ca $\mathrm{Ca}_{14} \mathrm{albi}_{11}$ - a new zintl phase from earth-abundant elements with a great potential for thermoelectric energy conversion," Mater. Today Adv. 7, 100094 (2020).

${ }^{17}$ J. Y. Chan, S. M. Kauzlarich, P. Klavins, R. N. Shelton, and D. J. Webb, "Colossal magnetoresistance in the transition-metal zintl compound $\mathrm{eu}_{14} \mathrm{mnsb}_{11}$," Chem. Mater. 9, 3132 (1997).

${ }^{18}$ W.-j. Tan, Y.-t. Liu, M. Zhu, T.-j. Zhu, X.-b. Zhao, X.-t. Tao, and S.-q. Xia, "Structure, magnetism, and thermoelectric properties of magnesium-containing antimonide zintl phases $\operatorname{sr}_{14}$ mgsb $_{11}$ and $e_{14}$ mgsb $_{11}$," Inorg. Chem. 56, 1646 (2017).

${ }^{19}$ E. S. Toberer, S. R. Brown, T. Ikeda, S. M. Kauzlarich, and G. Jeffrey Snyder, "High thermoelectric efficiency in lanthanum doped $\mathrm{yb}_{14} \mathrm{mnsb}_{11}$," Appl. Phys. Lett. 93, 062110 (2008).

${ }^{20}$ E. L. Kunz Wille, N. S. Grewal, S. K. Bux, and S. M. Kauzlarich, "Seebeck and figure of merit enhancement by rare earth doping in $\mathrm{yb}_{14-x} \mathrm{re}_{x} \mathrm{znsb}_{11}(\mathrm{x}=0.5)$," Materials 12, 731 (2019).

${ }^{21}$ E. S. Toberer, C. A. Cox, S. R. Brown, T. Ikeda, A. F. May, S. M. Kauzlarich, and G. J. Snyder, "Traversing the metal-insulator transition in a zintl phase: rational enhancement of thermoelectric efficiency in $\mathrm{yb}_{14} \mathrm{mn}_{1-x} \mathrm{al}_{x} \mathrm{sb}_{11}$," Adv. Funct. Mater. 18, 2795 (2008).

${ }^{22}$ I. Fisher, S. Bud'Ko, C. Song, P. Canfield, T. Ozawa, and S. M. Kauzlarich, "Yb $b_{14} z$ bb $_{11}$ : charge balance in zintl compounds as a route to intermediate yb valence," Phys. Rev. Lett. 
85, $1120(2000)$.

${ }^{23}$ J. P. Makongo, G. M. Darone, S.-Q. Xia, and S. Bobev, "Non-stoichiometric compositions arising from synergistic electronic and size effects. synthesis, crystal chemistry and electronic properties of $A_{14} \mathrm{~cd}_{1+x} P n_{11}$ compounds $(0 \leq \mathrm{x} \leq 0.3 ; A=\mathrm{sr}$, eu; $P n=\mathrm{as}, \mathrm{sb})$," J. Mater. Chem. C 3, 10388 (2015).

${ }^{24}$ R. Gallup, C. Fong, and S. M. Kauzlarich, "Bonding properties of calcium gallium arsenide, ca $_{14}$ gaas $_{11}$ : a compound containing discrete gaas 4 tetrahedra and a hypervalent as 3 polyatomic unit," Inorg. Chem. 31, 115 (1992).

${ }^{25}$ C. J. Perez, M. Wood, F. Ricci, G. Yu, T. Vo, S. K. Bux, G. Hautier, G.-M. Rignanese, G. J. Snyder, and S. M. Kauzlarich, "Discovery of multivalley fermi surface responsible for the high thermoelectric performance in $\mathrm{yb}_{14} \mathrm{mnsb}_{11}$ and $\mathrm{yb}_{14} \mathrm{mgsb}_{11}$," Science Advances 7, eabe9439 (2021).

${ }^{26}$ Y. Wang, Y.-J. Hu, S. A. Firdosy, K. E. Star, J.-P. Fleurial, V. A. Ravi, L.-Q. Chen, S.-L. Shang, and Z.-K. Liu, "First-principles calculations of lattice dynamics and thermodynamic properties for $\mathrm{yb}_{14} \mathrm{mnsb}_{11}, "$ J. Appl. Phys. 123, 045102 (2018).

${ }^{27} \mathrm{~J} . \mathrm{Xu}$ and $\mathrm{H}$. Kleinke, "Unusual sb-sb bonding in high temperature thermoelectric materials," J. Comput. Chem. 29, 2134 (2008).

${ }^{28}$ Y. Hu, K. Lee, and S. M. Kauzlarich, "Optimization of ca ${ }_{14} \mathrm{mgsb}_{11}$ through chemical substitutions on sb sites: Optimizing seebeck coefficient and resistivity simultaneously," Crystals 8, 211 (2018).

${ }^{29}$ S. M. Kauzlarich, Chemistry, Structure, and Bonding of Zintl Phases and Ions, 1st ed. (Wiley VCH, 1996).

${ }^{30}$ E. S. Toberer, C. A. Cox, S. R. Brown, T. Ikeda, A. F. May, S. M. Kauzlarich, and G. J. Snyder, "Traversing the metal-insulator transition in a zintl phase: Rational enhancement of thermoelectric efficiency in $\mathrm{yb}_{14} \mathrm{mn}_{1 x} \mathrm{al}_{x} \mathrm{sb}_{11}, "$ Adv. Funct. Mater. 18, 2795 (2008).

${ }^{31}$ E. S. Toberer, S. R. Brown, T. Ikeda, S. M. Kauzlarich, and G. Jeffrey Snyder, "High thermoelectric efficiency in lanthanum doped $\mathrm{yb}_{14}$ mnsb $_{11}, "$ Appl. Phys. Lett. 93, 062110 (2008), https://doi.org/10.1063/1.2970089.

${ }^{32}$ J. Xu and H. Kleinke, "Unusual sb-sb bonding in high temperature thermoelectric materials," J. Comput. Chem. 29, 2134 (2008).

${ }^{33}$ W. G. Zeier, J. Schmitt, G. Hautier, U. Aydemir, Z. M. Gibbs, C. Felser, and G. J. Snyder, "Engineering half-heusler thermoelectric materials using zintl chemistry," Nat. 
Rev. Mater. 1, 1 (2016).

${ }^{34}$ G. Kresse, "Ab initio molecular dynamics for liquid metals," J. Non-Cryst. Solids 192, $222(1995)$.

${ }^{35}$ G. Kresse and J. Furthmüller, "Efficiency of ab-initio total energy calculations for metals and semiconductors using a plane-wave basis set," Comp. Mater. Sci. 6, 15 (1996).

${ }^{36}$ G. Kresse and J. Furthmüller, "Efficient iterative schemes for ab initio total-energy calculations using a plane-wave basis set," Phys. Rev. B 54, 11169 (1996).

${ }^{37}$ S. Maintz, B. Eck, and R. Dronskowski, "Speeding up plane-wave electronic-structure calculations using graphics-processing units," Comput. Phys. Commun. 182, 1421 (2011).

${ }^{38}$ M. Hacene, A. Anciaux-Sedrakian, X. Rozanska, D. Klahr, T. Guignon, and P. FleuratLessard, "Accelerating vasp electronic structure calculations using graphic processing units," J. Comput. Chem. 33, 2581 (2012).

${ }^{39}$ M. Hutchinson and M. Widom, "Vasp on a gpu: Application to exact-exchange calculations of the stability of elemental boron," Comput. Phys. Commun. 183, 1422 (2012).

${ }^{40}$ P. E. Blöchl, "Projector augmented-wave method," Phys. Rev. B 50, 17953 (1994).

${ }^{41}$ G. Kresse and D. Joubert, "From ultrasoft pseudopotentials to the projector augmentedwave method," Phys. Rev. B 59, 1758 (1999).

${ }^{42}$ H. J. Monkhorst and J. D. Pack, "Special points for brillouin-zone integrations," Phys. Rev. B 13, 5188 (1976).

${ }^{43}$ P. E. Blöchl, O. Jepsen, and O. K. Andersen, "Improved tetrahedron method for brillouinzone integrations," Phys. Rev. B 49, 16223 (1994).

${ }^{44}$ T. A. Albright, J. K. Burdett, and M.-H. Whangbo, Orbital interactions in chemistry (John Wiley \& Sons, 2013).

${ }^{45}$ D. Sánchez-Portal, R. M. Martin, S. M. Kauzlarich, and W. Pickett, "Bonding, moment formation, and magnetic interactions in $\mathrm{ca}_{14} \mathrm{mnbi}_{11}$ and $\mathrm{ba}_{14} \mathrm{mnbi}_{11}$," Phys. Rev. B 65, 144414 (2002).

${ }^{46}$ M. Toriyama, M. Brod, and G. J. Snyder, "Chemical interpretation of charged point defects in semiconductors: A case study of $\operatorname{mg}_{2}$ si," ChemRxiv (2021), 10.33774/chemrxiv2021-729bm.

${ }^{47}$ W. Tan, Z. Wu, M. Zhu, J. Shen, T. Zhu, X. Zhao, B. Huang, X.-t. Tao, and S.-q. Xia, " $A_{14} \mathrm{mgbi}_{11}(A=\mathrm{ca}$, sr, eu): Magnesium bismuth based zintl phases as potential thermoelectric materials," Inorg. Chem. 56, 10576 (2017). 
${ }^{48}$ S. Baranets, G. M. Darone, and S. Bobev, "Synthesis and structure of $\operatorname{sr}_{14} \mathrm{Zn}_{1+x}$ as 11 and $\mathrm{eu}_{14} \mathrm{Zn}_{1+x} \mathrm{as}_{11}(\mathrm{x} \leq 0.5)$. new members of the family of pnictides isotypic with $\mathrm{ca}_{14} \mathrm{alsb}_{11}$, exhibiting a new type of structural disorder," J. Solid State Chem. 280, 120990 (2019).

${ }^{49} \mathrm{~S}$. Baranets and S. Bobev, "From the ternary phase $\mathrm{ca}_{14} \mathrm{Zn}_{1+\delta} \mathrm{sb}_{11}(\delta 0.4)$ to the quaternary solid solutions $\mathrm{ca}_{14-x} R E_{x} \mathrm{znsb}_{11}(R E=\mathrm{la}, \mathrm{nd}, \mathrm{sm}, \mathrm{gd}, \mathrm{x} 0.9)$. a tale of electron doping via rare-earth metal substitutions and the concomitant structural transformations," Inorg. Chem. 58, 8506 (2019).

${ }^{50}$ Y. Hu, S. K. Bux, J. H. Grebenkemper, and S. M. Kauzlarich, "The effect of light rare earth element substitution in $\mathrm{yb}_{14} \mathrm{mnsb}_{11}$ on thermoelectric properties," J. Mater. Chem. C 3, 10566 (2015). 